\title{
Avaliação de interpoladores espaciais para melhoria do mapeamento de atributos químicos do solo na cultura da banana no Estado do Tocantins, Brasil
}

Evaluation of spatial interpolators for improving the mapping of soil chemical attributes in banana cultivation in the Tocantins State, Brazil

Evaluación de interpoladores espaciales para mejorar el mapeo de atributos químicos del suelo en el cultivo de banano en el Estado de Tocantins, Brasil

Recebido: 04/01/2021 | Revisado: 05/01/2021 | Aceito: 07/01/2021 | Publicado: 08/01/2021

\author{
Virgilio Lourenço Silva Neto \\ ORCID: https://orcid.org/0000-0002-1004-0191 \\ Instituto Federal de Educação, Ciência e Tecnologia do Tocantins, Brasil \\ E-mail: virgilio.neto@ifto.edu.br \\ José Alberto Ferreira Cardoso \\ ORCID: https://orcid.org/0000-0002-7343-4614 \\ Instituto Federal de Educação, Ciência e Tecnologia do Tocantins, Brasil \\ E-mail: jose.ferreira@ifto.edu.br \\ Otacílio Silveira Junior \\ ORCID: https://orcid.org/0000-0002-0784-5335 \\ Instituto Federal de Educação, Ciência e Tecnologia do Tocantins, Brasil \\ E-mail: otacilio.junior@ifto.edu.br \\ Elismar Dias Batista \\ ORCID: https://orcid.org/0000-0002-4391-4238 \\ Instituto Federal de Educação, Ciência e Tecnologia do Tocantins, Brasil \\ E-mail: elismar.batista@ifto.edu.br \\ Amanda Marques Medrado Araújo \\ ORCID: https://orcid.org/0000-0001-8192-6179 \\ Universidade Estadual Paulista, Brasil \\ E-mail: amandamedradoifto@gmail.com \\ Ítalo Cordeiro Silva Lima \\ ORCID: https://orcid.org/0000-0001-6326-7974 \\ Instituto Federal de Educação, Ciência e Tecnologia do Tocantins, Brasil \\ E-mail: italo.lima@ifto.edu.br
}

\begin{abstract}
Resumo
O objetivo deste estudo foi avaliar a qualidade de diferentes interpoladores para a distribuição espacial dos atributos químicos do solo na cultura de banana. Foram coletadas amostras de solo em 10 pontos diferentes, nas profundidades de $0,0-0,20 \mathrm{~m}$ e $0,20-0,40 \mathrm{~m}$. Para espacialização dos atributos químicos, foram avaliados por meio da validação cruzada o desempenho dos interpoladores Krigagem Ordinária (KO) e Inverso da Potência da Distância (IPD), sendo que a KO teve os modelos esférico, exponencial e gaussiano avaliados, assim como o IPD teve o desempenho avaliado nos expoentes 2,3 e 5 . Verificou-se por meio da assimetria e curtose, valores entre 0 e 3 , indicando assim, a normalidade dos dados. Os menores coeficientes de variação foram encontrados para os atributos $\mathrm{pH}$ e V\% (profundidade de 0,00-0,20 m) e $\mathrm{Ca}^{2+}$ (profundidade de 0,20-0,40 m). Para todos os outros atributos, os coeficientes de variação encontrados foram acima de $20 \%$. Maiores valores apresentados foram para os atributos $\mathrm{Mg}^{2+}, \mathrm{K}, \mathrm{H}+\mathrm{Al}^{3+}$. Dentre os interpoladores escolhidos com melhor desempenho, pode-se afirmar que $20 \%$ dos resultados demonstram que o interpolador IPD-2 se sobressaiu, assim como o IPD-3. O IPD-5 foi o que apresentou melhor desempenho em $40 \%$ dos resultados. Os interpoladores geoestatísticos não tiveram um desempenho satisfatório, sendo que o interpolador KO modelo Esférico, em nenhum momento comportou-se com o melhor desempenho dentre todos os interpoladores testados. O interpolador KO modelo Exponencial teve o melhor desempenho em 15\% dos resultados, e o KO modelo Gaussiano se sobressaiu em apenas $5 \%$ dos resultados.
\end{abstract}

Palavras-chave: Agricultura de precisão; Geoestatística; IPD.

\begin{abstract}
The aim of this study is to evaluate the quality of different interpolators for the spatial distribution of soil chemical attributes in banana cultivation. Soil samples were collected at 10 different points, at depths of $0.0-0.20 \mathrm{~m}$ and 0.20 $0.40 \mathrm{~m}$. For spatialization of the chemical attributes of the soil, the performance of the Ordinary Kriging (KO) and Inverse Distance Power (IPD) interpolators were evaluated through cross validation, and the KO had the spherical, exponential and Gaussian models evaluated, as well as the IPD had its performance evaluated in exponents 2, 3 and 5 . It was found, through asymmetry and kurtosis, values between 0 and 3 , thus indicating the normality of the data. The
\end{abstract}


lowest coefficients of variation were found for the attributes $\mathrm{pH}$ and $\mathrm{V} \%$ (depth of $0.00-0.20 \mathrm{~m}$ ) and $\mathrm{Ca}^{2+}$ (depth of $0.20-0.40 \mathrm{~m}$ ). For all other attributes, the variation coefficients found were above $20 \%$. Higher values were presented for the attributes $\mathrm{Mg}^{2+}, \mathrm{K}, \mathrm{H}+\mathrm{Al}^{3+}$. Among the interpolators chosen with the best performance, it can be said that $20 \%$ of the results demonstrate that the IPD-2 interpolator stood out, as well as the IPD-3. The IPD-5 showed the best performance in $40 \%$ of the results. The geostatistical interpolators did not perform satisfactorily, and the interpolator KO Spherical model, at no time behaved with the best performance among all the interpolators tested. The KO model Exponential interpolator had the best performance in $15 \%$ of the results, and the KO Gaussian model excelled in only $5 \%$ of the results.

Keywords: Precision agriculture; Geostatistics; IDW.

\section{Resumen}

El objetivo de este estudio fue evaluar la calidad de diferentes interpoladores para la distribución espacial de los atributos químicos del suelo en cultivos de banano. Fueran recolectadas muestras de suelo en 10 puntos diferentes, a una profundidad de 0.0-0.20 m y 0.20-0.40 m. Para la espacialización de atributos químicos se evaluó el desempeño de los interpoladores Ordinary Kriging (KO) y Distance Power Inverse (IPD) usando validación cruzada, teniendo el KO evaluado los modelos esférico, exponencial y gaussiano, así como el IPD el desempeño se evaluó en los exponentes 2, 3 y 5 . Los valores entre 0 y 3 se verificaron mediante asimetría y curtosis, indicando así la normalidad de los datos. Los coeficientes de variación más bajos se encontraron para los atributos pH y V\% (profundidad de 0.00$0.20 \mathrm{~m}$ ) y Ca2+ (profundidad de 0.20-0.40 m). Para el resto de los atributos, los coeficientes de variación encontrados fueron superiores al 20\%. Las mayores variaciones fueron encontradas en los atributos $\mathrm{K}, \mathrm{H}+\mathrm{Al} 3+$. Entre los interpoladores elegidos con mejor desempeño, se puede decir que el $20 \%$ de los resultados demuestran que se destacó el interpolador IPD-2, así como el IPD-3. El IPD-5 mostró el mejor desempeño en el 40\% de los resultados. Los interpoladores geoestadísticos no tuvieron desempeño satisfactorio, y el interpolador $\mathrm{KO}$ modelo esférico, en ningún momento se comportó con el mejor desempeño entre todos los interpoladores probados. El interpolador exponencial del modelo KO tuvo el mejor desempeño en el 15\% de los resultados, y el modelo Gaussiano KO sobresalió en solo el $5 \%$ de los resultados.

Palabras clave: Agricultura de precisión; Geoestadística; IPD.

\section{Introdução}

A intensificação do uso da tecnologia no setor agropecuário vem contribuindo para o crescente desenvolvimento das atividades do setor, permitindo o aumento gradativo da produtividade. Avanços tecnológicos na agropecuária têm mostrado a importância de se medir a variação espacial e temporal de propriedades que afetam o rendimento das culturas, com o objetivo de otimizar o aproveitamento de recursos e diminuir custos (Carvalho et al., 2002). Segundo Oliveira et al. (2017), através dos avanços tecnológicos na agropecuária, especialistas têm notado, cada vez mais, que os diversos setores da agricultura não podem ser tratados de maneira homogênea no que diz respeito à medição de variáveis nas áreas agrícolas.

Experimentos de campo são, em sua maioria, divididos em parcelas ou áreas relativamente pequenas amostradas aleatoriamente. Contudo, ao considerar as parcelas experimentais uniformes quanto aos seus atributos, mesmo em pequenas áreas, pode-se interpretar erroneamente as respostas obtidas às questões existentes, pois a hipótese de ocorrência de dependência espacial será ignorada (Grego \& Vieira, 2005).

Angelico (2006) afirma que no passado, as análises estatísticas de dados consideravam as variáveis aleatórias independentes entre si, ou seja, supunham que as observações vizinhas não exerciam influências umas sobre as outras. Porém, fenômenos naturais apresentam-se frequentemente com certa estruturação entre vizinhos. Assim, pode-se dizer que as variações não ocorrem ao acaso, apresentando certo grau de dependência espacial. Considera-se indispensável a caracterização da variabilidade espacial dos atributos químicos e físicos do solo por meio de amostragem capaz de representar tais variações (Bottega et al., 2013). Este estudo, especificamente, considera interpoladores geoestatísticos para avaliar a existência de dependência espacial, comparando com interpoladores determinísticos como o Inverso da Potência da distância - IPD.

Diversos estudos sobre a variabilidade espacial dos atributos do solo já foram realizados. Recentemente, Almeida \& Guimarães (2016b), verificaram o comportamento espacial do pH e dos macronutrientes, para um melhor planejamento adequado do uso de corretivos e fertilizantes na Fazenda Brasil, município de Araguari, MG. Almeida \& Guimarães (2016a) também realizaram estudos no intuito de avaliar a dependência espacial dos atributos químicos do solo por meio da associação 
de técnicas geoestatísticas e de análise multivariada, visando contribuir com a tomada de decisões que visem à produtividade e sustentabilidade da cafeicultura. Bernardi et al. (2011) realizaram o mapeamento da variabilidade espacial dos atributos químicos e físicos do solo e elaborar mapas de necessidade de aplicação de insumos de forma localizada em área de pasto rotacionado manejado intensivamente, na Embrapa Pecuária Sudeste, em São Carlos, SP. Souza et al. (2010) caracterizaram a variabilidade espacial de atributos físicos e o teor de matéria orgânica de um Latossolo Vermelho eutroférrico gibbsítico (sob Basalto) e Latossolo Vermelho distrófico caulinítico (sob Arenito) nas profundidades de 0,0-0,2m e 0,2-0,4m, em áreas sob cultivo de cana-de-açúcar no nordeste do Estado de São Paulo (SP), nos Municípios de Guariba (SP) e Jaboticabal (SP). Sousa et al. (2015), avaliar as modificações impostas sobre a densidade, porosidade e consequentemente resistência a penetração do solo, em duas áreas distintas uma de Cerrado nativo e outra um cultivo de eucalipto na região sul do Estado do Tocantins.

O objetivo deste estudo foi avaliar a qualidade de diferentes interpoladores para a distribuição espacial dos atributos do solo em uma área de cultivo irrigado da banana (7,8 ha), dentro do perímetro irrigado do Manuel Alves, região Sudeste do Tocantins.

\section{Metodologia}

Este trabalho constitui um estudo de caso, de natureza quantitativa, baseada em um grande volume de dados numéricos tratados por meio de técnicas geoestatísticas com intuito de geração de equações matemáticas que possibilitem a espacialização dos dados obtidos (Pereira et al., 2018).

A área de estudo encontra-se na bacia do Rio Manuel Alves da Natividade, que pertence ao sistema hidrográfico do rio Tocantins (margem direita), com uma área de drenagem de 14.934,93 km², contemplando municípios 11 municípios, que abrangem uma área de aproximadamente $22.576 \mathrm{~km}^{2}$ (SEPLAN, 2012). Esta área representa grande importância para o Estado do Tocantins, pois nela se localiza o Projeto Manuel Alves na região sudeste, é um dos grandes projetos de irrigação do país, pela dimensão de seu reservatório de águas e pela extensão da área irrigável, por microaspersão, gotejamento e aspersão convencional, onde se pratica a cultura de abacaxi, banana, coco, mamão, maracujá e melancia, além de mandioca, milho, tomate, abóbora cabutiá, cana-de-açúcar, goiaba e pupunha (Prolo et al., 2019).

Figura 1 - Localização da área de estudo na Bacia do Rio Manuel Alves da Natividade.

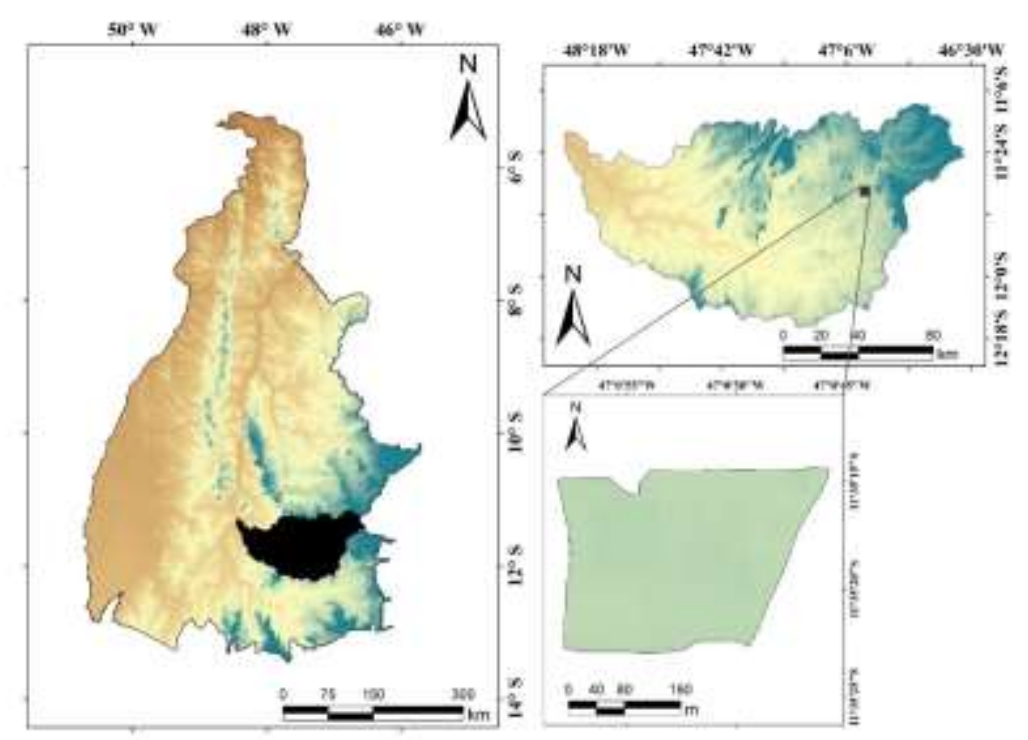

Nota: Conforme a Figura 1 é possível verificar que a área de estudo está localizada na bacia do Rio Manuel Alves da Natividade, situada na porção sudeste do estado do Tocantins. Fonte: Autores. 
$\mathrm{A}$ área de estudo está compreendida na região climática $\mathrm{C} 2 \mathrm{wA}^{\prime} \mathrm{a}$ ' - Subúmido, com deficiência de água moderada no inverno, megatérmico. É importante considerar que embora o tipo climático desta região seja subúmido, devido ao excedente hídrico de dezembro a março, o período seco, principalmente entre maio a setembro, há um considerável deficit hídrico (Souza et al., 2019).

O experimento foi conduzido em uma área localizada no sudeste do Tocantins, na bacia do rio Manuel Alves da Natividade, equivalente a 7,8 ha onde é praticado o cultivo irrigado da banana (Musa spp), variedade prata (5 anos de idade). Foram coletadas amostras deformadas de solo em 10 pontos diferentes, nas profundidades de 0,0-0,20 m e 0,20-0,40 m.

Para espacialização dos atributos químicos do solo, foram avaliados o desempenho dos interpoladores Krigagem Ordinária (KO) e Inverso da Potência da Distância (IPD), sendo que a KO teve os modelos esférico, exponencial e gaussiano avaliados, assim como o IPD teve o desempenho avaliado nos expoentes 2,3 e 5.

Uma etapa essencial da análise geoestatística consiste no ajuste do modelo de semivariograma teórico, obtendo-se valores para os parâmetros que estruturam tais modelos (efeito pepita, contribuição e alcance). Neste estudo, foram avaliados os modelos de semivariograma esférico, exponencial e gaussiano, tendo sido aplicado o método de ajuste dos Mínimos Quadrados Ponderados (MQP). Nas Equações 1, 2 e 3 estão apresentados os modelos de semivariograma esférico, exponencial e gaussiano, respectivamente.

$$
\begin{aligned}
& V(h)=C_{0}+C_{1} \cdot\left[\frac{3}{2} \cdot\left(\frac{h}{a}\right)-\frac{1}{2} \cdot\left(\frac{h}{a}\right)^{3}\right], \text { se } 0<h<a ; \boldsymbol{V}(h)=C 0+C 1 ; h \geq a \\
& V(h)=C_{0}+C_{1} \cdot\left[1-\exp \left(\frac{-3 \cdot h}{a}\right)\right], \quad \text { se } 0<h<a ; \boldsymbol{V}(h)=C 0+C 1 ; h>a \\
& V(h)=C_{0}+C_{1} \cdot\left\{1-\exp \left[-3 \cdot\left(\frac{h}{a}\right)^{2}\right]\right\}, \text { se } 0<h<a ; \boldsymbol{V}(h)=C 0+C 1 ; h>a
\end{aligned}
$$

Sendo, $V(h)$ a semivariância, $\mathrm{C}_{1}$ a contribuição do semivariograma (diferença entre o patamar e o efeito pepita), $a$ o alcance, $\mathrm{C}_{0}$ o efeito pepita e $\boldsymbol{h}$ a distância entre os pares de pontos.

Com o intuito de analisar a estrutura de dependência espacial dos modelos de semivariograma ajustados foi calculado o grau de dependência espacial (GD), sendo obtido conforme Cambardella et al. (1994) por:

$$
G D=\left(\frac{C_{1}}{C_{0}+C_{1}}\right) 100
$$

Sobre o GD, pode-se adotar a seguinte classificação: GD < 25\% (fraco), entre 25 e $75 \%$ (moderado) e > 75 \% (forte) (Cambardella et al., 1994).

Objetivando identificar o modelo de semivariograma com melhor desempenho, foi realizada a validação cruzada. Foi quantificado o Erro Médio Percentual Absoluto (EMPA), possibilitando apontar o modelo que produziu o menor erro de estimativa, e que será definido para o mapeamento de cada evento em análise. Procedimento análogo foi adotado em diversos 
estudos de mesma natureza, com destaque para o trabalho desenvolvido por Melo et al. (2005). O EMPA, em \%, foi calculado utilizando a seguinte expressão:

$$
E M P A=\frac{1}{N} \cdot \sum_{i=1}^{n}\left|\frac{O b s_{i}-E s t_{i}}{O b s_{i}}\right| \cdot 100
$$

Em que, $n$ é o número de postos pluviométricos, Obs $s_{i}$ a precipitação observada para o posto i e $E s t_{i}$ é a precipitação estimada por krigagem para a posição do posto $\boldsymbol{i}$.

O viés estatístico (Bias) foi calculado pela média da diferença entre a série simulada e a observada, indicando o erro sistemático do interpolador (subestimativa ou superestimativa). O valor do Bias corresponde ao percentual de viés das variáveis estimadas com relação às variáveis observadas. O Bias, em \%, foi obtido pela Equação 11, de acordo com Liew et al. (2007):

$$
B I A S=\sum_{k=1, n}^{n}\left(Q \mid k o b s-Q_{k s i m}\right)(100) / \sum_{k=1, n}^{n}(Q \mid k o b s)
$$

Os valores foram classificados da seguinte maneira: $|\mathrm{Bias}|<10 \%$, considerado muito bom; entre $10 \%$ e $<15 \%$, bom; entre $15 \%$ e $<25 \%$, satisfatório e $|\operatorname{Bias}| \geq 25 \%$, o modelo é considerado inadequado (Liew et al., 2007).

Também foi testado o interpolador inverso da potência da distância (IPD), tendo sido avaliados os expoentes 2, 3 e 5. A formulação do interpolador IPD encontra-se descrita na Eq. 7 (Cecilio \& Pruski, 2003). A justificativa para aplicação do IPD encontra-se na inviabilidade de utilização de interpoladores geoestatísticos, pois os mapeamentos foram desenvolvidos a partir de dados de apenas 10 localidades do estado do Tocantins, não sendo suficiente para modelar a estrutura de dependência espacial da variável em análise.

$$
X_{P}=\frac{\sum_{i=1}^{n}\left(\frac{X_{i}}{d_{i}^{m}}\right)}{\sum_{i=1}^{n}\left(\frac{1}{d_{i}^{m}}\right)}
$$

Em que $\mathrm{X}_{\mathrm{p}}$ corresponde à variável interpolada na posição $p, X_{i}$ é a PMP conhecida nos $i$ pontos vizinhos, di é a distância euclidiana da estação $i$ ao ponto $p$ a ser estimado, $m$ é o expoente da distância euclidiana e $n$ é o número de estações com dados de PMP disponíveis.

Foi adotado o sistema de Coordenadas SIRGAS 2000 para a elaboração dos mapas.

\section{Resultados e Discussão}

Na Tabela 1 são apresentados os valores referentes aos resultados da análise descritiva das variáveis químicas do solo avaliadas na área de estudo. 
Tabela 1 - Estatística descritiva do Potencial hidrogeniônico $(\mathrm{pH})$, Fósforo (P), Potássio (K), Cálcio $\left(\mathrm{Ca}^{2+}\right)$, Magnésio $\left(\mathrm{Mg}^{2+}\right)$, Acidez potencial $\left(\mathrm{H}^{+} \mathrm{Al}^{3+}\right)$, Soma das Bases $(\mathrm{SB})$, capacidade de troca catiônica (CTC) e saturação por base (V\%) e matéria orgânica (MO), nas profundidades de 0,00 a 0,20 $\mathrm{m}$ em área de cultivo irrigado de banana.

Profundidade 0,00 a $0,20 \mathrm{~m}$

\begin{tabular}{|c|c|c|c|c|c|c|c|}
\hline & Média & Mediana & DP & $\mathrm{CV}(\%)$ & KS & Assimetria & Curtose \\
\hline $\mathrm{pH}$ & 6,31 & 6,60 & 0,62 & 9,90 & 0,28 & $-0,65$ & $-1,52$ \\
\hline $\mathrm{P}\left(\mathrm{mg} \cdot \mathrm{dm}^{-3}\right)$ & 10,87 & 11,66 & 5,46 & 50,24 & 0,20 & $-0,30$ & $-1,68$ \\
\hline $\mathrm{K}\left(\mathrm{cmol}_{\mathrm{c} .} \mathrm{dm}^{-3}\right)$ & 42,10 & 34,00 & 17,32 & 41,15 & 0,21 & 1,53 & 0,93 \\
\hline $\mathrm{Ca}^{2+}\left(\mathrm{cmol}_{\mathrm{c}} \cdot \mathrm{dm}^{-3}\right)$ & 2,21 & 2,20 & 0,74 & 33,35 & 0,18 & $-0,14$ & $-1,59$ \\
\hline $\mathrm{Mg}^{2+}\left(\mathrm{cmol}_{\mathrm{c}} \cdot \mathrm{dm}^{-3}\right)$ & 0,94 & 0,90 & 0,47 & 50,45 & 0,11 & 0,30 & $-1,44$ \\
\hline $\mathrm{H}+\mathrm{Al}^{3+}\left(\mathrm{cmol}_{\mathrm{c}} \cdot \mathrm{dm}^{-3}\right)$ & 3,04 & 2,89 & 0,84 & 27,60 & 0,18 & 0,55 & $-0,18$ \\
\hline $\mathrm{SB}\left(\mathrm{cmol}_{\left.\mathrm{c} \cdot \mathrm{dm}^{-3}\right)}\right.$ & 3,26 & 3,45 & 1,07 & 32,72 & 0,16 & $-0,15$ & $-1,34$ \\
\hline CTC $\left(\mathrm{cmol}_{\mathrm{c} .} \mathrm{dm}^{-3}\right)$ & 6,29 & 6,52 & 1,64 & 26,05 & 0,14 & $-0,34$ & $-1,38$ \\
\hline $\mathrm{V} \%$ & 51,21 & 52,37 & 7,48 & 14,60 & 0,18 & $-0,09$ & $-1,13$ \\
\hline \multirow[t]{2}{*}{$\mathrm{MO}(\%)$} & 1,63 & 1,61 & 0,39 & 23,98 & 0,14 & 0,33 & $-1,28$ \\
\hline & & & & & & & Continua... \\
\hline
\end{tabular}

Profundidade 0,20 a $0,40 \mathrm{~m}$

\begin{tabular}{|c|c|c|c|c|c|c|c|}
\hline & Média & Mediana & DP & $\mathrm{CV}(\%)$ & KS & Assimetria & Curtose \\
\hline $\mathrm{pH}$ & 6,63 & 6,65 & 0,33 & 5,03 & 0,14 & 0,09 & $\overline{-1,40}$ \\
\hline $\mathrm{P}\left(\mathrm{mg} \cdot \mathrm{dm}^{-3}\right)$ & 3,92 & 3,08 & 1,94 & 49,53 & 0,17 & 0,84 & $-0,79$ \\
\hline $\mathrm{K}\left(\mathrm{cmol}_{\mathrm{c} .} \mathrm{dm}^{-3}\right)$ & 29,60 & 23,00 & 16,26 & 54,94 & 0,17 & 1,71 & 1,70 \\
\hline $\mathrm{Ca}^{2+}\left(\mathrm{cmol}_{c} \cdot \mathrm{dm}^{-3}\right)$ & 1,87 & 1,80 & 0,35 & 18,70 & 0,10 & 0,63 & $-1,12$ \\
\hline $\mathrm{Mg}^{2+}\left(\mathrm{cmol}_{\mathrm{c}} \cdot \mathrm{dm}^{-3}\right)$ & 1,52 & 1,50 & 0,60 & 39,45 & 0,11 & 0,02 & $-1,43$ \\
\hline $\mathrm{H}+\mathrm{Al}^{3+}\left(\mathrm{cmol}_{\mathrm{c} . \mathrm{dm}^{-3}}\right)$ & 2,15 & 2,31 & 1,08 & 50,38 & 0,21 & $-0,56$ & $-0,02$ \\
\hline $\mathrm{SB}\left(\mathrm{cmol}_{\mathrm{c} \cdot} \cdot \mathrm{dm}^{-3}\right)$ & 3,47 & 3,28 & 0,90 & 26,07 & 0,11 & 0,38 & $-1,25$ \\
\hline CTC $\left(\mathrm{cmol}_{\mathrm{c} .} \mathrm{dm}^{-3}\right)$ & 5,61 & 5,60 & 1,71 & 30,45 & 0,12 & 0,08 & $-0,61$ \\
\hline $\mathrm{V} \%$ & 64,35 & 57,13 & 16,57 & 25,75 & 0,14 & 1,54 & 1,24 \\
\hline $\mathrm{MO}(\%)$ & 1,39 & 1,34 & 0,31 & 22,00 & 0,11 & 0,03 & $-1,31$ \\
\hline
\end{tabular}

DP = Desvio Padrão; CV(\%) = Coeficiente de Variação, KS = Teste de Kolmogorov-Smirnov significativo a 5\%. Fonte: Autores.

A Tabela 1 contém as informações sobre os atributos do solo em cultivo irrigado da banana, nas profundidades de 0,00 a 0,20 m e 0,20 a 0,40 m, com ênfase na média, mediana, Desvio Padrão, Coeficiente de Variação e teste de KolmogorovSmirnov significativo a $5 \%$, assimetria e curtose.

É possível verificar por meio da assimetria e curtose, valores entre 0 e 3, indicando assim, a normalidade dos dados (Carvalho et al., 2002; Soares et al., 2018). Com exceção do K, as variáveis apresentaram distribuição de frequência com média e mediana semelhantes, podendo ser consideradas como simétricas (Oliveira et al, 2017). Quanto ao teste de KomogorvSmirnov com nível de 5\% de significância, indicando que todos os atributos seguem uma distribuição normal. 
Os menores coeficientes de variação foram encontrados para os atributos $\mathrm{pH}$ e V\% (profundidade de 0,00-0,20 m) e $\mathrm{Ca}^{2+}$ (profundidade de 0,20-0,40 m). Para todos os outros atributos, os coeficientes de variação encontrados foram acima de 20\%. Maiores valores apresentados foram para os atributos $\mathrm{Mg}^{2+}, \mathrm{K}, \mathrm{H}+\mathrm{Al}^{3+}$. Valores elevados de coeficiente de variação são muito comuns em estudos dessa natureza. Segundo Carvalho et al. (2002), uma explicação plausível para esta variabilidade seria o fato de se ter feito uma amostragem simples nos pontos amostrados. Segundo o autor, com amostragens compostas, seria muito provável encontrar menores variabilidades. Porém, a variação na pequena distância pode ser uma informação importante principalmente para os elementos que têm pouca mobilidade. Resultados semelhantes foram encontrados em estudos realizados por Oliveira et al. (2009) e Soares et al., (2018).

A dependência espacial classificada como "Forte" de acordo com Cambardella et al., (1994) foi verificada em apenas 28 situações de 60 possíveis análises por meio do interpolador geoestatístico Krigagem Ordinária, considerando os modelos Esférico, Exponencial e Gaussiano. Em função disso, pode-se inferir que o desempenho destes interpoladores não é satisfatório na grande maioria das situações analisadas. A explicação para isso, pode estar na baixa quantidade de amostras coletadas. Segundo Silva Neto et al. (2019), neste caso, justifica-se a aplicação do IPD devido à inviabilidade de utilização de interpoladores geoestatísticos, uma vez que a maioria dos mapeamentos foram desenvolvidos com dados de apenas 10 pontos de coleta.

Na Tabela 2 estão apresentados os valores do Erro Médio Percentual Absoluto (EMPA), considerado para apontar o interpolador com melhor desempenho por meio da técnica da validação cruzada. 
Tabela 2 - Erro Médio Percentual Absoluto - EMPA (\%) dos interpoladores testados para o mapeamento dos atributos do solo sob o cultivo irrigado da banana.

\begin{tabular}{|c|c|c|c|c|c|c|}
\hline \multicolumn{7}{|c|}{ Profundidade 0,00 a $0,20 \mathrm{~m}$} \\
\hline & IPD-2 & IPD-3 & IPD-5 & $\mathrm{KO}(1)$ & $\mathrm{KO}(2)$ & $\mathrm{KO}(3)$ \\
\hline $\mathrm{pH}$ & 7,93 & $7,90 *$ & 8,19 & 8,12 & 8,06 & 8,19 \\
\hline $\mathrm{P}\left(\mathrm{mg} \cdot \mathrm{dm}^{-3}\right)$ & 82,01 & 80,66 & 79,11 & 77,87 & 80,18 & $14,26 *$ \\
\hline $\mathrm{K}\left(\mathrm{cmol}_{\mathrm{c} .} \mathrm{dm}^{-3}\right)$ & 24,19 & $13,30 *$ & 24,03 & 24,72 & 24,55 & 25,13 \\
\hline $\mathrm{Ca}^{2+}\left(\mathrm{cmol}_{\mathrm{c}} \cdot \mathrm{dm}^{-3}\right)$ & 32,46 & $13,86^{*}$ & 31,29 & 36,31 & 34,36 & 37,05 \\
\hline $\mathrm{Mg}^{2+}\left(\mathrm{cmol}_{\mathrm{c}} \cdot \mathrm{dm}^{-3}\right)$ & 57,21 & 57,93 & 58,58 & 62,09 & $9,12 *$ & 63,67 \\
\hline $\mathrm{H}+\mathrm{Al}^{3+}\left(\mathrm{cmol}_{\mathrm{c}} \cdot \mathrm{dm}^{-3}\right)$ & 25,00 & 25,72 & 26,31 & 8,67 & $8,54 *$ & 8,52 \\
\hline $\mathrm{SB}\left(\mathrm{cmol}_{\mathrm{c}} \cdot \mathrm{dm}^{-3}\right)$ & 29,35 & 28,74 & $8,33 *$ & 31,77 & 31,46 & 33,97 \\
\hline CTC $\left(\mathrm{cmol}_{\mathrm{c} \cdot} \cdot \mathrm{dm}^{-3}\right)$ & 22,29 & $12,78 *$ & 22,76 & 24,25 & 23,27 & 26,00 \\
\hline $\mathrm{V} \%$ & 13,32 & 13,38 & $13,24 *$ & 13,30 & 23,21 & 13,38 \\
\hline $\mathrm{MO}(\%)$ & 19,21 & 18,05 & $7,32 *$ & 22,71 & 22,71 & 22,71 \\
\hline \multicolumn{7}{|c|}{ Profundidade 0,20 a $0,40 \mathrm{~m}$} \\
\hline & IPD-2 & IPD-3 & IPD-5 & $\mathrm{KO}(1)$ & $\mathrm{KO}(2)$ & $\mathrm{KO}(3)$ \\
\hline $\mathrm{pH}$ & $5,53 *$ & 5,93 & 6,27 & 4,62 & 4,62 & 4,62 \\
\hline $\mathrm{P}\left(\mathrm{mg} \cdot \mathrm{dm}^{-3}\right)$ & 43,04 & 41,01 & $9,19 *$ & 45,34 & 45,72 & 45,88 \\
\hline $\mathrm{K}\left(\mathrm{cmol}_{\mathrm{c}} \cdot \mathrm{dm}^{-3}\right)$ & 46,35 & 47,86 & $7,21 *$ & 40,07 & 40,07 & 40,07 \\
\hline $\mathrm{Ca}^{2+}\left(\mathrm{cmol}_{\mathrm{c}} \cdot \mathrm{dm}^{-3}\right)$ & 10,49 & 9,90 & $9,33 *$ & 11,75 & 10,93 & 18,05 \\
\hline $\mathrm{Mg}^{2+}\left(\mathrm{cmol}_{\mathrm{c} \cdot} \cdot \mathrm{dm}^{-3}\right)$ & 43,09 & 43,94 & 44,88 & 45,69 & $12,41 *$ & 47,65 \\
\hline $\mathrm{H}+\mathrm{Al}^{3+}\left(\mathrm{cmol}_{\mathrm{c} \cdot \mathrm{dm}^{-3}}\right)$ & $17,54 *$ & 181,09 & 189,78 & 174,11 & 177,16 & 170,06 \\
\hline SB $\left(\mathrm{cmol}_{\mathrm{c}} \cdot \mathrm{dm}^{-3}\right)$ & $9,22 *$ & 19,96 & 20,39 & 20,69 & 19,61 & 23,31 \\
\hline CTC $\left(\mathrm{cmol}_{\mathrm{c} \cdot} \cdot \mathrm{dm}^{-3}\right)$ & 27,09 & 27,03 & $11,76^{*}$ & 29,69 & 28,19 & 30,78 \\
\hline $\mathrm{V} \%$ & $12,06^{*}$ & 23,18 & 24,38 & 20,43 & 20,60 & 20,29 \\
\hline MO (\%) & 21,71 & 21,68 & $10,95^{*}$ & 21,97 & 21,97 & 21,97 \\
\hline
\end{tabular}

IPD-2 = Inverso da Potência da Distância com expoente 2, IPD-3 = Inverso da Potência da Distância com expoente 3, IPD-5 = Inverso da Potência da Distância com expoente 5, KO (1) = Krigagem Ordinária - modelo Esférico, KO (2) = Krigagem Ordinária - modelo Exponencial e KO (3) = Krigagem Ordinária - modelo Gaussiano. * Interpolador com melhor desempenho. Fonte: Autores.

Na Tabela 2, foram destacados em negrito e com (*) o interpolador com melhor desempenho, a partir da observação do EMPA, comparando os resultados dos interpoladores IPD com expoente 2, 3 e 5 e a Krigagem Ordinária, nos modelos esférico, exponencial e gaussiano para cada atributo do solo.

Considerando os interpoladores escolhidos com melhor desempenho com base na validação cruzada, pode-se afirmar que em $20 \%$ dos resultados o interpolador IPD-2 se sobressaiu, sendo utilizado para mapear $\mathrm{pH}, \mathrm{H}+\mathrm{Al}{ }^{3+}, \mathrm{SB}$ e $\mathrm{V} \%$ na profundidade de 0,20-0,40 m, assim como o IPD-3, escolhidos para geração dos mapas pH, K, $\mathrm{Ca}^{2+}$ e CTC na profundidade de 0,00-0,20 m. Neste universo, o IPD-5 foi o que apresentou melhor desempenho em 40\% dos resultados, usado para gerar os mapas de SB, V\% e MO na profundidade de 0,00-0,20 m e P, K, $\mathrm{Ca}^{2+}$, CTC e MO na profundidade de 0,20-0,40 m. 
Como já mencionado, os interpoladores geoestatísticos não tiveram um desempenho satisfatório, sendo que a KO modelo Esférico, em nenhum momento comportou-se com o melhor desempenho dentre todos os interpoladores testados. O interpolador $\mathrm{KO}$ modelo Exponencial teve o melhor desempenho em 15\% dos resultados, gerando os mapas de $\mathrm{Mg}^{2+} \mathrm{e} \mathrm{H}+$ $\mathrm{Al}^{3+}$ na profundidade de 0,00-0,20 m e $\mathrm{Mg}^{2+}$ na profundidade de 0,20-0,40 m, e o KO modelo Gaussiano se sobressaiu em apenas $5 \%$ dos resultados, utilizado para gerar o mapa de $\mathrm{P}$ na profundidade de 0,00 a $0,20 \mathrm{~m}$.

A Figura 2 apresenta os mapas gerados para cada atributo químico do solo por meio do interpolado com o melhor desempenho com base na validação cruzada. 
Figura 2 - Mapas da variabilidade espacial dos atributos do solo $\mathrm{pH}, \mathrm{P}, \mathrm{K}, \mathrm{Ca}, \mathrm{Mg}, \mathrm{H}+\mathrm{Al}^{3+}$, Soma de Bases (SB), Capacidade de Troca Catiônica (CTC), saturação por base (V) e matéria orgânica (MO), sob o cultivo irrigado da banana, obtidos pelo interpolador com melhor desempenho.
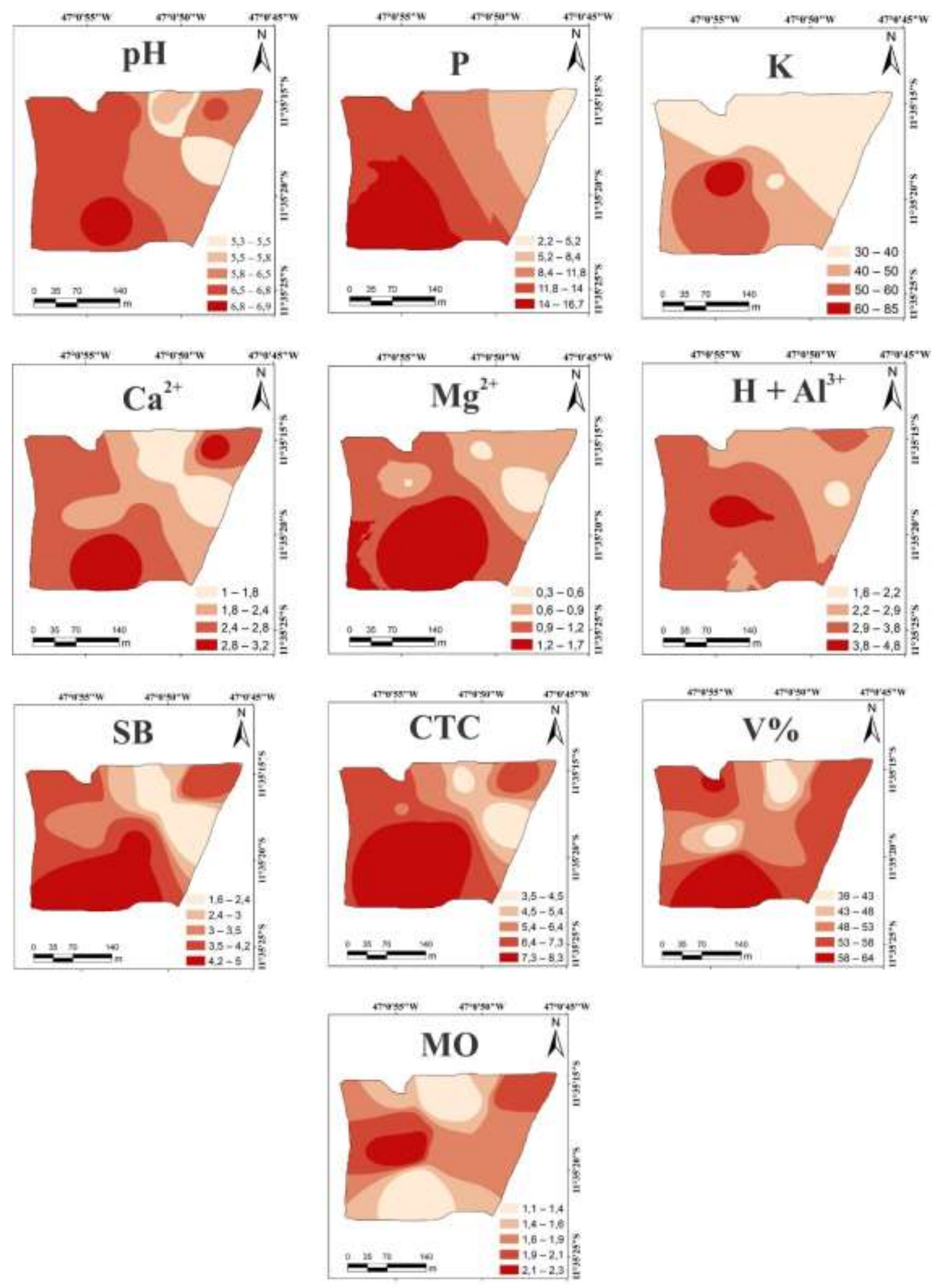

Fonte: Autores.

Por meio da observação da Figura 2, observa-se que os menores valores do pH encontram-se a nordeste da área de estudo, aumentando gradativamente na direção sudoeste. Para os atributos, P, CTC, $\mathrm{Mg}^{2+}, \mathrm{SB}$ e V\%, as maiores concentrações estão situadas ao sul da área de estudo. O atributo MO apresentou os maiores valores na porção centro-oeste da área de estudo. 
Praticamente todos atributos apresentaram comportamento de redução dos valores interpolados em direção a porção nordeste da área pesquisada.

Ao analisar os valores médio de $\mathrm{pH}, 6,47$, foram enquadrados na faixa considerada como acidez fraca para classificação química, enquanto na classificação agronômica como adequada (Alvarez et al., 1999). Segundo Borges et al., 2000, o limite inferior considerada adequada para a cultura da banana varia de 5,5 a 6,5. Portanto, os valores médios de pH observado no presente estudo encontra-se adequado, e associado com os valores de saturação por base $\mathrm{V}, \mathrm{pH}, \mathrm{Ca}$ e $\mathrm{Mg}$, o solo amostrado não demonstra necessidade de recomendação para uso de corretivos.

De acordo com os resultados da análise química para fins de interpretação de fertilidade de solo, os atributos químicos do solo foram classificados como teores médio para todas as variáveis estudadas (Alvarez et al., 1999). A utilização de fertilizantes para o suprimento das demandas nutricionais da cultura podem ter contribuído para os teores médios dos macronutrientes observados.

Segundo Roquin (2010), a saturação por base superior a 50\% apresentada no solo sob bananeira irrigada o caracteriza como eutrófico, ou seja, considera-se um solo fértil, enquanto na área de referência caracteriza-se como distrófico, pois a saturação por base apresentada é inferior a 50\%. Além disso, observa-se nos mapas gerados (Figura 2), uma heterogeneidade dos atributos químicos dentro da área estudada, podendo levar uma recomendação inadequada do manejo da fertilidade pelo método tradicional, uso da média dos valores para fins de recomendações para uso de fertilizante, sugerindo assim, o uso da geoestatística para o manejo da fertilidade.

\section{Conclusão}

É possível verificar por meio da assimetria e curtose, valores entre 0 e 3, indicando assim, a normalidade dos dados. Com exceção do K, as variáveis apresentaram distribuição de frequência com média e mediana semelhantes, podendo ser consideradas como simétricas. Quanto ao teste de Komogorv-Smirnov com nível de 5\% de siginificância, indicando que todos os atributos seguem uma distribuição normal.

Os interpoladores geoestatísticos não apresentaram desempenho satisfatório, sem dependência espacial na maioria dos casos analisados, devido ao número reduzido de pontos de coleta.

O IPD-5 apresentou o melhor desempenho em 40\% dos resultados obtidos e considerado apenas a natureza do interpolador foi possível identificar que em $80 \%$ dos resultados o IPD se sobressaiu em relação à Krigagem Ordinária.

É importante destacar que este estudo baseou-se amostras deformadas de solo em 10 pontos diferentes, nas profundidades de 0,0-0,20 m e 0,20-0,40 m. Assim, é de grande importância que trabalhos futuros disponham de um número de pontos de coleta substancialmente maior, tanto para aprimorar a análise do desempenho dos interpoladores quanto para compreensão dos atributos do solo nas condições observadas.

\section{Agradecimentos}

Ao Instituto Federal de Educação, Ciências e Tecnologia do Tocantins pela concessão de bolsa de pesquisa ao

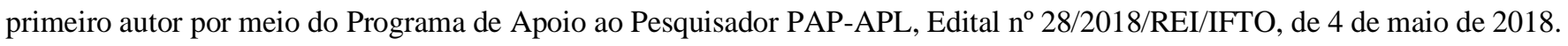

\section{Referências}

Almeida, L. S., \& Guimarães, E. C. (2016a). Geoestatística e análise fatorial exploratória para representação espacial de atributos químicos do solo, na cafeicultura. Coffee Science, 11(2), 195-203.

Almeida, L. S., \& Guimarães, E. C. (2016b). Geoestatística como ferramenta para o manejo sustentável da fertilidade do solo na cafeicultura. Revista de Ciências Agrárias/Amazonian Journal of Agricultural and Environmental Sciences, 59(4), 370-377. 
Alvarez, V. V. H., Novais, R. F., Barros, N. F., Cantarutti, R. B., \& Lopes, A. S. (1999) Interpretações dos resultados das análises de solos. In: Ribeiro, A. C., Guimarães, P. T. G., Alvarez, V. V. H. (Eds.). Recomendações para o uso de corretivos e fertilizantes em Minas Gerais - $5^{a}$ aproximação. Viçosa: CFSEMG.

Angelico, J. C. (2006). Desempenho da co-krigagem na determinação da variabilidade de atributos do solo. Revista Brasileira de Ciência do Solo, 30(6), 931936.

Bernardi, A. C. C., Bettiol, G. M., Inamasu, R. Y., \& Rabelo, L. (2011). Variabilidade espacial de propriedades do solo em pastagem manejada intensivamente em São Carlos, SP. In: Inamasu, R. Y., Naime, J. M., Resende, A. V., Bassoi, L. H., Bernardi, A. C. C. (Ed.). Agricultura de precisão: um novo olhar. São Carlos: Embrapa Instrumentação.

Bottega, E. L., Queiroz, D. M., Pinto, F. A. C., \& Souza, C. M. A. (2013). Variabilidade espacial de atributos do solo em sistema de semeadura direta com rotação de culturas no cerrado brasileiro. Revista Ciência Agronômica, 44(1), 1-9.

Borges, A. L., \& Oliveira, A. M. G. (2000). Nutrição, calagem e adubação. In: Cordeiro, Z. J. M. (Org.) Banana: produção - aspectos técnicos. Brasília: EMBRAPA.

Cambardella, C., Moorman, T. B., Novak, J. M., Parkin, T. B., Karlen, D. L., Turco, R. F., \& Konopka, A. E. (1994). Field-scale variability of soil properties in central Iowa soils. Soil Science Society of America Journal, 58(5), 1501-1511.

Carvalho, J. R. P., Da Silveira, P. M., \& Vieira, S.R. (2002). Geoestatística na determinação da variabilidade espacial de características químicas do solo sob diferentes preparos. Pesquisa Agropecuária Brasileira, 37(8), 1151-1159.

Cecilio, R. A., \& Pruski, F. F. (2003). Interpolação dos parâmetros da equação de chuva da equação de chuvas intensas com uso do inverso de potências da distância. Revista Brasileira de Engenharia Agrícola e Ambiental, 7(3), 501-504.

Grego, C. R., \& Vieira, S. R. (2005). Variabilidade espacial de propriedades físicas do solo em uma parcela experimental. Revista Brasileira de Ciência do Solo, 29(2), 169-177.

Liew, M.W., Veith, T. L., Bosch, D. D., \& Arnold, J. G. (2007). Suitability of SWAT for the conservation effects assessment project: Comparison on USDA agricultural research service watersheds. Journal of Hydrologic Engineering, 12(2), 173-189.

Mello, J. M., Batista, J. L. F., Ribeiro Júnior, P. J., \& Oliveira, M. S. (2005). Ajuste e seleção de modelos espaciais de semivariograma visando à estimativa volumétrica de Eucalyptus grandis. Scientia Forestalis, 69, 25-37.

Oliveira, P. C. G., Farias, P. R. S., Lima, H. V., Fernandes, A. R., Oliveira, F. A., \& Pita, J. D. (2009). Variabilidade espacial de propriedades químicas do solo e da produtividade de citros na Amazônia Oriental. Revista Brasileira de Engenharia Agrícola e Ambiental, 13(6), 708-715.

Oliveira, R. B.; Silva, S. A., Souza, G. S., Passos, R. R., Prezotti, L. C., \& Lima, J. S. S. (2017). Análise espacial de variáveis indicadoras de fertilidade do solo sob cultivo de cafeeiro conilon. Anais. X Encontro Latino Americano de Iniciação Científica e VI Encontro Latino Americano de Pós-Graduação Universidade do Vale do Paraíba, 2787-2790.

Pereira A. S., Shitsuka, D. M., Parreira, F. J., \& Shitsuka, R. (2018). Metodologia da pesquisa científica. UFSM. https://repositorio.ufsm.br/bitstream/handle/1/15824/Lic_Computacao_Metodologia-Pesquisa-Cientifica.pdf?sequence=1 .

Prolo, T. T., Silva Neto, V. L., Araujo, P. H. H., Rego, P. L., Silveira Junior, O., Carmo, E. L. (2019). Precipitação Máxima Diária Anual na Bacia do Rio Manuel Alves da Natividade. In: (Ed.). Competência técnica e responsabilidade social e ambiental nas ciências agrárias. Ponta Grossa: Atena Editora.

Ronquim, C. C. (2010). Conceitos de fertilidade do solo e manejo adequado para as regiões tropicais. Campinas: Embrapa Monitoramento por Satélite.

SEPLAN. (2012). Secretária de Planejamento. Atlas do Tocantins: Subsídios ao Planejamento da Gestão Territorial. Superintendência de Planejamento e Gestão central de Políticas Públicas. Diretoria de Zoneamento Ecológico-Econômico - DZE. Organizado por. Borges, R. S. T.; Dias, R. R.; Sousa, P. A. B. (orgs). 6. ed. rev. atua. Palmas: Seplan, p.80.

Silva Neto, V. L., Bonfim, D. D., Prolo, T. T., Carmo, E. L., Silveira Junior, O., Viola, M. R., Silva, L. L. (2019). Mapeamento da Precipitação Máxima Provável na bacia do rio Manuel Alves da Natividade. In: Rodrigues, T. D. A. \& Neto, J. L. (Ed.). Competência Técnica e Responsabilidade Social e Ambiental nas Ciências Agrárias. Ponta Grossa: Atena Editora.

Soares, M. D. R., Campos, M. C. C., Oliveira, I. A., Cunha, J. M., Souza, Z. M., Aquino, R. E., Silva, D. P., \& Silva, J. F. (2018). Variabilidade espacial dos atributos do solo sob agrofloresta na região de Humaitá, AM. Gaia Scientia, 12(1), 33-41.

Sousa, F. H. M., Silva Neto, V. L., Patriota, J., N., Sales, N. I. S., Neves, C. O. M., \& Lima, S. O. (2015). Atributos físicos do solo em diferentes coberturas florestais. Amazônia: Ciência \& Desenvimento, 11(21), 1-14.

Sousa, F. H. M., Viola, M. R., Avanzi, J. C., Giongo, M., \& Vieira Filho, M. (2019). Thornthwaite's Climate Regionalization for the State of Tocantins, Brazil. Floresta, 49(4), 783-792.

Souza, Z. M., Marques Júnior, J., \& Pereira, G. T. (2010). Geoestatística e atributos do solo em áreas cultivadas com cana-de-açúcar. Ciência Rural, 40(1), 4856 . 